\title{
Occupational Therapy in Integrative Visual Agnosia and Memory Disorder: A Case Report
}

\author{
Masahiro Tanaka \\ Department of Rehabilitation, Faculty of Health Science, Nihon Fukushi University
}

\begin{abstract}
Visual agnosia is a rare symptom in which the patient cannot recognize objects by visual perception. We report a case of integrative visual agnosia and memory disorder. A 70-year-old, right-handed man was diagnosed with cardioembolic stroke affecting the proximal left posterior cerebral artery. He had a short-term memory disorder. He could not name and explain how to use objects, or read characters, but could discriminate between objects to some extent and draw copies of simple figures. His recognition by tactile sense was comparatively preserved. He needed complete assistance with his life in the hospital ward. We provided him with training using preserved tactile sensation by errorless learning. We arranged the environment around him to promote his memory of the position of objects and to avoid misuse of daily objects. Improvement in the visual agnosia and memory disorder was slight. He became independent with his life in the hospital ward. However, he needed assistance in a new environment, such as an unfamiliar lavatory. The results suggest that a patient with visual agnosia may become independent early by appropriate training, despite insufficient visual perception and memory function. In our patient, the intervention period was too short to generalize the effects of training. Additionally, the memory disorder might have become an obstacle for generalization. Further improvement in visual perception, independence in daily activities, and quality of life, will need continuous support.
\end{abstract}

Keywords: visual agnosia, memory disorder, occupational therapy

(Asian J Occup Ther 17: 27-30, 2021)

\section{Introduction}

Visual agnosia is a form of cognitive deficit in which the patient cannot recognize objects by visual perception. Visual agnosia is traditionally divided into two types, apperceptive visual agnosia and associative visual agnosia [1]. Humphreys advocated another type, integrative visual agnosia [2].

Integrative agnosia is thought to be an impairment of the perceptual integration that shapes elements into coherent wholes [2]. A patient with integrative visual agnosia can discriminate and draw copies of objects they see, but they cannot recognize them [2].

Visual agnosia is a rare symptom with few reports about the rehabilitation process. Here, we describe a patient with integrative visual agnosia and memory dis-

Received: 26 May 2020, Accepted: 22 December 2020

Corresponding to: Masahiro Tanaka, Department of Rehabilitation, Faculty of Health Science, Nihon Fukushi University, 26-2, Higashihaemi-chou, Handa, Aichi, Japan

e-mail: tmasahir@n-fukushi.ac.jp

(C)2021 Japanese Association of Occupational Therapists order induced by cardioembolic stroke.

The publication of this report was explained to the patient and his family with a written document, and informed consent was obtained from a family member.

\section{Case Report}

The patient was a 70-year-old, right-handed man who complained of headache and vision problems when he presented to our hospital. He was not paralyzed and did not have a sensory disorder. He had right homonymous hemianopsia, slight aphasia, memory disorder, and visual agnosia. His language was fluent, and he could have daily conversations with mild difficulty in finding words. He was diagnosed with cardioembolic stroke at the proximal left posterior cerebral artery by magnetic resonance imaging (Fig. 1).

He had a short-term memory disorder where he could not remember recent events but could partially learn repeated information. His visual perception was generally impaired and Visual Perception Test for Agnosia (VPTA) [3] results are shown in Fig. 2 left half. He could not name or explain how to use objects, or read 


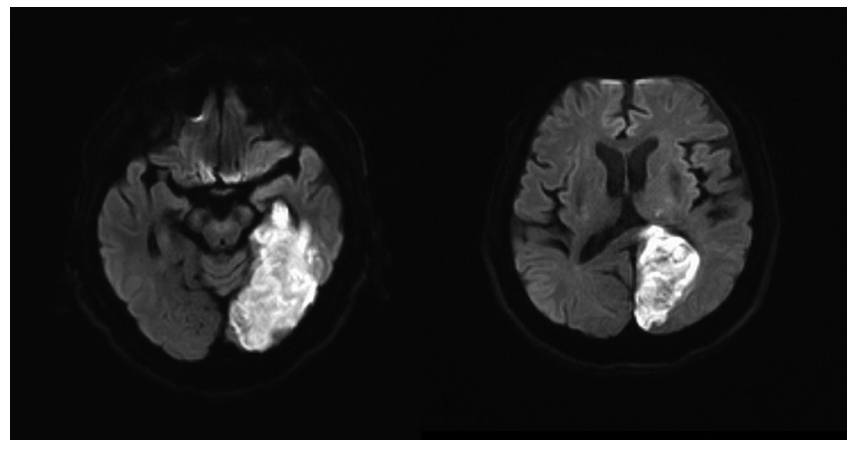

Fig. 1. The diffusion weighted image of brain

characters, but could partially discriminate objects and draw copies of simple figures after a long time. Recognition by tactile sense was comparatively preserved, as he could occasionally use objects properly when he touched them. He needed assistance and guidance with eating and personal hygiene since he could not recognize or use the appropriate objects, and with using the toilet because he could not reach the lavatory and wandered in the ward. Before the onset of stroke, he was completely independent in his daily life.

We started occupational therapy on the second day of admission. We focused on his preserved tactile function and that he could learn information with repetition. We provided him with therapy so he could perform activities of independent daily living by compensating with tactile sensation. We helped him learn how to eat, maintain personal hygiene, and toileting by repeatedly practicing in the hospital ward. We made him touch objects required for eating and personal hygiene, and learn strategies to aid recognition (Fig. 3 left). Further, we identified the position of objects needed for eating and personal hygiene at the table to promote memory and avoid misuse (Fig. 3 middle). For toileting, we made him touch the toilet bowl, toilet paper, and flush button repeatedly so he could recognize and use them by tactile sensation (Fig. 3 right). We identified the location of the toilet early in his therapy so the effect of therapy could be generalized to an early period in his hospital stay. We used errorless learning so whenever he made a mistake, we would stop as soon as possible and guide him to the correct way. His symptoms and assistance required were described to the nursing staff so he could continue his practice. We provided occupational therapy, physical therapy, and speech and language therapy 5 days a week.

His word finding difficulties improved, while the memory disorder remained. He was able to roughly recall daily events and practice the contents during therapy. His VPTA score improved (Fig. 2 right half), although visual agnosia persisted and other parameters were unchanged. He could independently eat, maintain personal hygiene, and recognize objects on the table without touching. He was able to reach the predetermined lavatory and excrete without requiring tactile sensation for recognition or assistance. While he became independent in his daily life in the limited environment of the hospital ward, assistance was needed in new environments such as an unfamiliar lavatory.

The patient was transferred to a rehabilitation hospital 36 days after initial admission for further treatment.

\section{Discussion}

Based on the medical evaluation and symptoms, the patient was considered to have integrative visual agnosia, and his damaged lesion corresponded with previous studies of integrative visual agnosia $[2,4]$.

While there are few studies about rehabilitation of patients with visual agnosia, compensatory strategies are beneficial [5]. Further, errorless learning is an effective approach for patients with memory disorders [6]. Based on the patient's spontaneous functional recovery, we used a compensatory approach and errorless learning to improve his independence in daily life. Our results suggest that patients with visual agnosia can become independent early through appropriate training, despite impaired visual perception and memory function.

However, the patient needed assistance in a new environment. Zihl reported a patient with integrative visual agnosia who trained for a visual search task and could generalize the effect to untrained tasks [7]. In contrast, Behrmann reported a patient who trained for a visual recognition task and could generalize to other tasks [8]. The current intervention period was limited to an acute phase, which could be considered too short to generalize the effect of training on his long-term recovery. Memory disorders may also be an obstacle for generalization in a new environment.

Few reports exist on the improvement in daily object recognition during long-term follow-ups $[9,10]$. While our patient became independent in his daily life in the hospital ward, he needed further improvement. After discharge from the rehabilitation hospital, continuous support will be needed to improve his visual perception, independence in daily activities, and quality of life.

\section{Conclusion}

We have described a patient with integrative visual agnosia and memory disorder. The patient became independent early in his hospital ward stay by using preserved tactile function training. While the effects of this training did not generalize to a new environment, 

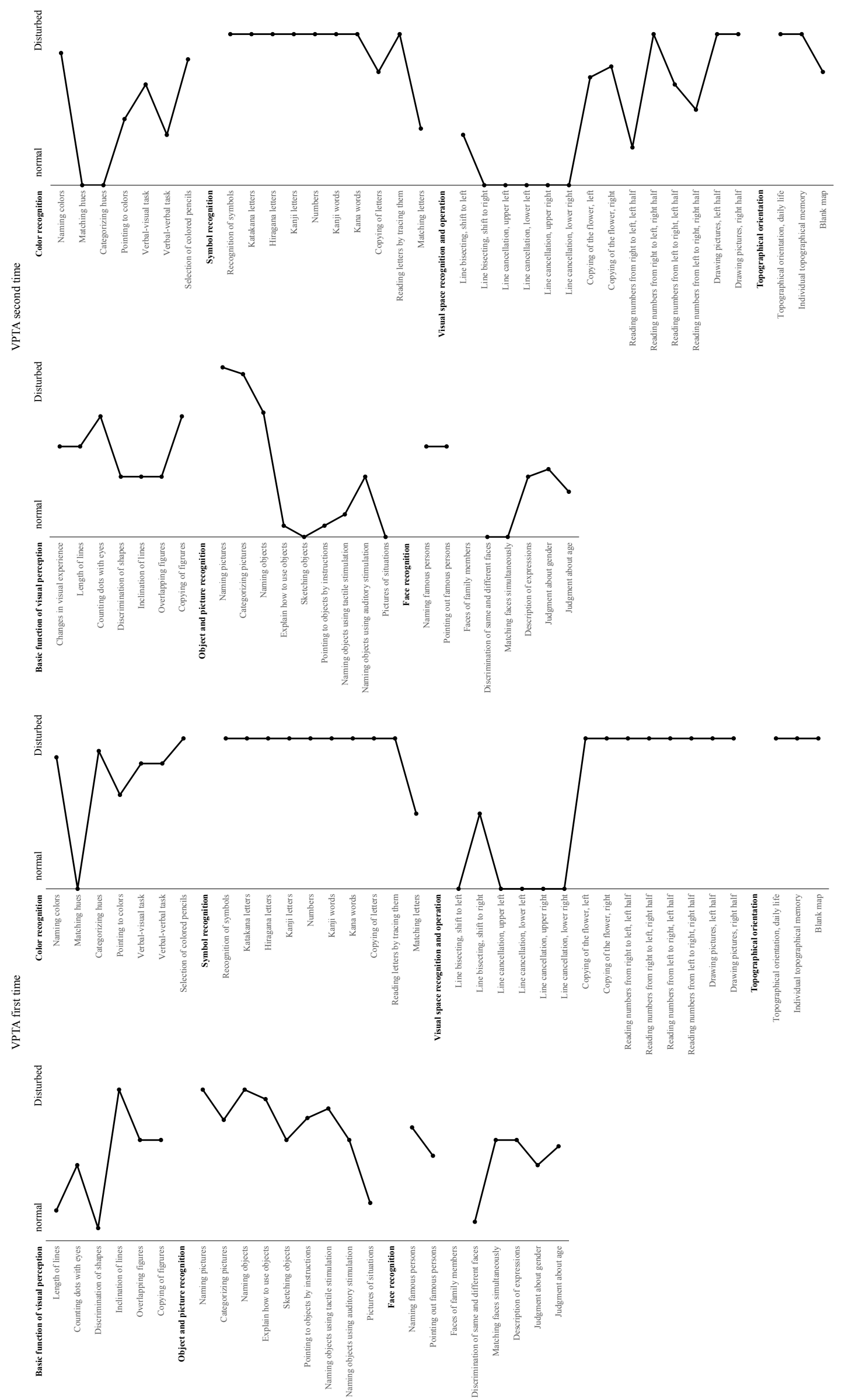

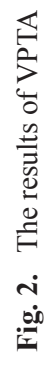



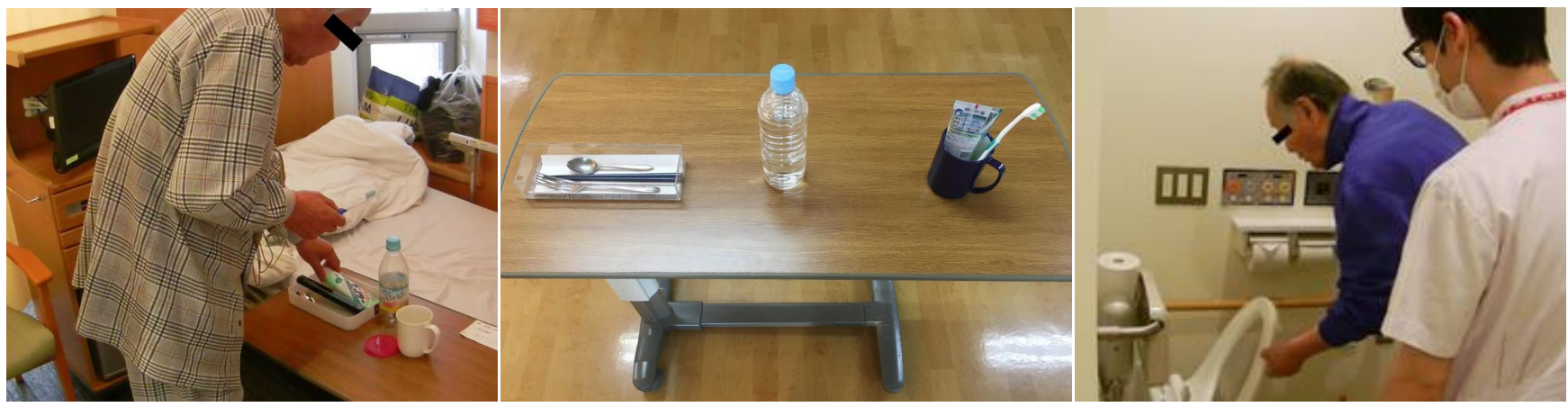

Fig. 2. The scene of occupational therapy and environmental arrangement

this could be due to the short intervention period and his memory disorder.

\section{Declaration of Conflicting Interests}

The author declares that there is no conflict of interest.

\section{Funding}

The author received no financial support for the research, authorship, and/or publication of this article.

\section{References}

[1] Lissauer H. Ein Fall von Seelenblindheit nebst einem Beitrage zur Theorie derselben. Archiv für Psychiatrie und Nervenkrankheiten. 1890; 21(2): 222-70.

[2] Riddoch MJ, Humphreys GW. A case of integrative visual agnosia. Brain. 1987;110(Pt 6): 1431-62.

[3] Japanese Society of Aphasiology. Visual Perception Test of Agnosia. Tokyo: Shinko Igaku Shuppan; 1997 (in
Japanese).

[4] Hirayama K, Suzuki Y, Inoue K. Integrative visual (object) agnosia. Clinical Neuroscience. 2012; 30(10): 1090-1 (in Japanese).

[5] Heutink J, Indorf DL, Cordes C. The neuropsychological rehabilitation of visual agnosia and Balint's syndrome. Neuropsychol Rehabil. 2019; 29(10): 1489-508.

[6] Jean L, Bergeron M-È, Thivierge S, Simard M. Cognitive Intervention Programs for Individuals With Mild Cognitive Impairment: Systematic Review of the Literature. Am J Geriatr Psychiatry. 2010; 18(4): 281-96.

[7] Zihl J. Rehabilitation of Visual Disorders After Brain Injury, 2nd Edition. 2011.

[8] Behrmann M, Marotta J, Gauthier I, Tarr MJ, McKeeff TJ. Behavioral change and its neural correlates in visual agnosia after expertise training. J Cogn Neurosci. 2005; 17(4): 554-68.

[9] Rubens AB, Benson D. Associative visual agnosia. Arch Neurol. 1971; 24(4): 305-16.

[10] Wilson BA, Davidoff J. Partial recovery from visual object agnosia: a 10 year follow-up study. Cortex 1993; 29(3): 529-42. 